\title{
Evaluation and Validation of Diagnostic Tests for Guiding Therapeutic Decisions
}

\author{
Paul Landais ${ }^{1 \star}$, Valérie Méresse ${ }^{2 \star}$, Jean-Claude Ghislain ${ }^{3}$ and the participants in Round Table $N^{\circ} 4$, Giens \\ $X X I V^{\star \star}$
}

1 Hôpital Necker, Paris, France

2 F Hoffmann-La Roche Ltd, Basel, Suisse

3 Afssaps, Saint-Denis, France

Text received 15 october 2008; accepted 20 november 2008

\section{Keywords:}

theranostics;

drug;

diagnostic test;

development;

recommendations

\begin{abstract}
The term "theranostics" characterizes a particular combination of the coupled use of a drug therapy with a diagnostic test (called "companion diagnostics"). The diagnostic test marks out the technical means used to identify a biomarker which allows for adjusting the use of the new drug.

Theranostics promise a significant step forward for the use of personalized medicine, better tailored for patients in terms of efficacy and tolerance. The development process of theranostics is complex since it requires a proper phasing from development to reimbursement, through a collaborative and controlled approach.

The task force's recommendations should form the basis for reflection and action amongst the different players in the field of the development of theranostics.

All stages of the system meeting heterogeneous and asynchronous rules should be harmonized to allow for simultaneous availability of, and access to the drug and test. Nevertheless, it is necessary to simplify the regulations to enable a better adaptation of theranostics to the speed of innovation.
\end{abstract}

\section{Theranostics}

The term "theranostics" characterizes a particular combination of the coupled use of a drug therapy together with a diagnostic test (occasionally called "companion diagnostics").

To be more specific, the diagnostic test marks out the technical mean used to identify a biomarker. According to the definition put forward by the National Institute of Health, a biomarker is "a characteristic that is objectively measured and evaluated as an indicator of normal biological processes, pathogenic processes, or pharmacological responses to a therapeutic intervention". [1]

The result of the diagnostic test allows for adjusting the use of the new drug according to three modalities:

- selection of a specific target population. It will be made up of patients whose response to the biomarker allows assuming

^ Both authors equally contributed to the work.

$\star \star$ For a list of participants, see the end of the article. that their tolerance and/or their response to the new drug will be good;

- determination of the dosage of the new drug particular for an individual;

- monitoring and controlling the specific response of the patient to the retained treatment.

Theranostics give new insight in a more targeted and individualized application of new treatments.

Theranostics can be distinguished from the classical combination of a drug and a diagnostic test (for example, the couple: measurement of glycaemia and prescription of an anti-diabetic drug) by at least two facts:

- the simultaneous and integrated development of the new diagnostic test together with the new drug that can be tailored accordingly,

- market authorization should account for the coupled combination of the diagnostic test together with the new drug. 


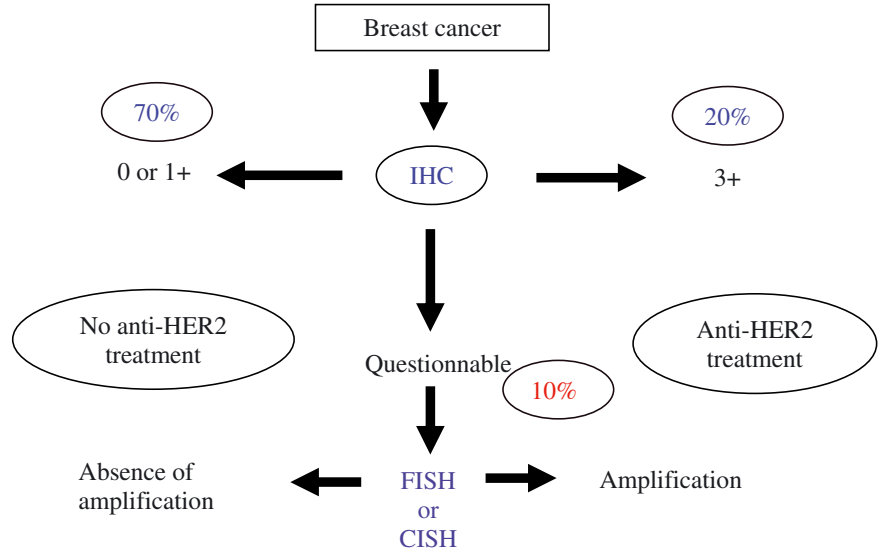

Fig. 1. Breast cancer and the strategy for the use of targeted anti-HER2 treatment according to the HER2 status evaluated by immuno-histochemistry (IHC) and in situ hybridization 5FISH); example of trastuzumab as adjuvant therapy.

There is however one exception to the latter proposal. In some cases, a new diagnostic test for a new biomarker might indeed still be developed after the marketing authorization has been granted for the associated drug.

\section{Examples}

The theranostic strategy can be illustrated at the hand of 2 examples of a "couple" composed of a drug and its companion test.

\section{1. Treatment targeting the HER2 receptor (trastuzumab, lapatinib|}

HER2 belongs to the family of "Human Epidermal Receptors" (HER). It is an important regulation factor for the growth and differentiation of normal cells. Around $15 \%$ of breast cancers are associated with an over-expression of HER2 which is assessed through immunohistochemistry (IHC) (over-expression rated " +3 "), and/or gene amplification using in situ hybridation either by fluorescence (FISH) or chromogene (CISH). Patients with breast cancers overexpressing HER2 have a worse prognosis than patients who do not overexpress the gene; the risk of relapse is increased and the overall survival time is shorter. Trastuzumab (Herceptin ${ }^{\circledR}$ ) is a humanized monoclonal antibody that binds specifically to the extracellular domain of the protein HER2. This antibody was developed for the treatment of metastatic breast cancer - and randomized clinical trials have demonstrated both its efficacy in terms of survival and good tolerance. ${ }^{[2-4]}$ It has also been shown in case of adjuvant therapy for breast cancer, when trastuzumab was administered alone or in combination with various chemotherapy regimens. ${ }^{[5]}$
Only patients over-expressing HER 2 (score +3 in IHC) or with an amplification of the gene (FISH/CISH+), are eligible for trastuzumab therapy. In case of a tumor presenting with an IHC score of +2 , a FISH or CISH is needed as a complement to assess whether the patient can indeed receive trastuzumab. In these cases, the antibody will be prescribed only if amplification is present. The breast cancer treatment algorithm based on trastuzumab will thus depend on the overexpression of HER2 oncogene assessed either by IHC (hyperexpression of the protein on the membrane), or by FISH (or CISH) (amplification of the oncogene HER2) [figure 1] in order to favour a therapy targeting HER2 receptors (e.g. treatment with trastuzumab with adjuvant therapy). The IHC technique is the most widely spread screening method for detecting an overexpression of HER2, because on the one hand it is the least expensive, and on the other hand, in France, it is presently the only technique which allows reimbursement from the NHS. For the test to be reliable, it must be calibrated according to the FISH or CISH technique. In France, under the aegis of the study group for immune-histochemical prognostic factors in breast cancer (GEFPICS), a quality control protocol ("FISH 2002") was designed to analyze the concordance of IHC and FISH in a large number of metastatic primitive breast tumors $(n=1500)$ by French pathological units. The main outcome of this study was to evaluate the concordance between the IHC and FISH techniques. It also aimed at demonstrating the impact of calibrating the IHC technique on the results of the FISH technique. In addition, a quality assurance policy has been set up with technical testing and interpretation developed by the French Association for Quality Assurance in Pathology (AFAQAP) and through workshops. ${ }^{[6]}$

The recent approval of lapatinib is another example of identification of a very specific target population (Lapatinib Procedure $N^{\circ}$. EMEA/H/C/795 Doc.Ref.: EMEA/302222/2008).

\subsection{Cetuximab and test evaluating the oncogenic KRAS mutation status}

Cetuximab just obtained a marketing authorization as firstline therapy for colorectal cancer depending on the KRAS gene mutation status. The following is written in the new summary of product characteristics (SPC) of cetuximab: “....Erbitux ${ }^{\circledR}$ is indicated for the treatment of patients with metastatic colorectal cancer expressing a wild-type KRAS gene associated with the epidermal growth factor receptor (EGFR), either in combination with chemotherapy, or as monotherapy after failure of a treatment composed of oxaliplatin and irinotecan, or in case of intolerance to irinotecan, ...". In fact, only the subset of patients who do not reveal the mutation of KRAS gene is likely to benefit from a treatment with cetuximab, since the mutation confers a very specific resistance to cetuximab. ${ }^{[7]}$ Cetuximab has already 
been launched on the market when the identification of KRAS gene mutations was discovered as a marker of resistance to antiEGFR antibody. ${ }^{[8]}$ At first the European authorities accepted the test since the panitumumab pivotal study demonstrated that this test had a significant positive predictive value for the effectiveness of the drug as third-line monotherapy. ${ }^{[9]}$ After this initial work, the test has been imposed and thereafter it demonstrated its interest as first-line therapy. ${ }^{[10]}$ The market authorization of cetuximab has been modified for all the therapeutic lines afterwards. The detection of KRAS gene mutations is based on a PCR (Polymerase Chain Reaction) reaction and on the identification of various mutations by means of sequencing, micro-sequencing, or allelic discrimination.

The importance of KRAS mutation as predictive factor of the response to cetuximab has been demonstrated in a recent article. ${ }^{[11]}$ An analysis of KRAS mutation and its association with the survival rate was performed on a sample of 394 metastatic colorectal tumors obtained from 572 patients $(68.9 \%)$ who were randomized to receive either cetuximab combined with the best supportive care, or the best supportive care alone. KRAS mutation status ( $42.3 \%$ of cases) was negatively correlated to the efficacy of cetuximab either for the interaction of KRAS mutation with the overall survival $(\mathrm{P}=0.01)$ or on progression-free survival $(\mathrm{P}<0.001)$, respectively. Patients with a tumour bearing wild-type KRAS mutation benefited significantly from treatment with cetuximab for both overall survival (median $9.5 \mathrm{vs}$. 4.8 months, $\mathrm{P}<0.001$ ) and progression-free survival (median 3.7 months vs. 1.9 months, $\mathrm{P}<0.001$ ), whereas patients with tumour bearing KRAS mutation did not benefit from cetuximab therapy. KRAS mutation did not appear to confer a worse prognosis in terms of overall survival in the group of patients treated by supportive care alone. This publication highlights the need to use a diagnostic test in all patients presenting with a metastatic colon cancer. This test will assess a biomarker (KRAS mutation) in a standardized and validated way in order to select the subset of patients bearing the KRAS mutation (42\%) who will not benefit from cetuximab. ${ }^{[12]}$

\subsection{Dosage-adjustment according to serum concentration levels of the medication (pharmacological treatment monitoringl}

One benefit of therapeutic targeting is the opportunity for tailored individual dosage adjustment. Tyrosine kinase inhibitors are concerned since it was demonstrated that the therapeutic response to imatinib in patients with chronic myeloid leukaemia was influenced by the serum concentrations of the medication. ${ }^{[13,14]}$ Similarly, the serum antibody concentrations have an influence on the efficacy, as is demonstrated in patients with malignant
non-Hodgkin's lymphoma treated with rituximab. ${ }^{[15,16]}$ However, there are still no recommendations for individual dosage adjustment based on blood levels.

\subsection{Predictive factors of side effects}

Diagnostic tests should also play a role in identifying patients at risk of side effects. A recent study demonstrated that hypersensitivity reactions noted in $3 \%$ of patients treated with cetuximab (an antibody anti-EGFR), might be linked, in some patients, to the pre-existence of $\operatorname{IgE}$ (immunoglobulin) oriented against a certain sugar present in this biodrug. ${ }^{[17]}$ The potential interest of searching for these IgE's (anti- $\alpha$-gal antibodies) prior to starting this monoclonal antibody would be worth investigating.

\section{Recommendations}

\section{1. Synchronising the development: diagnostic test and drug}

The relevance of the diagnostic test appears at various stages in the development of the drug as illustrated on figure 2.

The task force reports a lack of synchronization in the assessment and timely disposal of the couple "diagnostic test and drug": a lack of synchronization between the development of the drug and the diagnostic test (the procedures for the drug are usually faster); management by distinct regulatory bodies for marketing and for reimbursement requirements in the community.

The development procedures for the "test-drug" combination should be well structured from the outset and validated as early as possible. It is advised to perform a double analytical and clinical validation of the diagnostic test (nota bene: these recommendations should be transposable to an in vivo measure - e.g. functional imaging techniques). Evaluation of the "test/drug" couple should be encouraged in a prospective rather than a deferred way. One of the challenges of such a combination of test and drug, derives from the fact that it might require only a simple and single test on the one hand but also possibly a combination of multiple tests (for example a set of gene biomarkers) on the other hand. Any such tests could be either invasive or not (e.g., biopsy or blood samples) and should refer to specific regulations rather than to distinct ones as it is presently the case. Moreover, the simultaneous development of both test and drug typically requires a coupling with back and forth technical and knowledge processes that reflect the complexity of the implementation.

The qualification processes of a biomarker should be followed within this framework and according to the guidelines of the European Medicines Agency (EMEA) [in final stages]. 


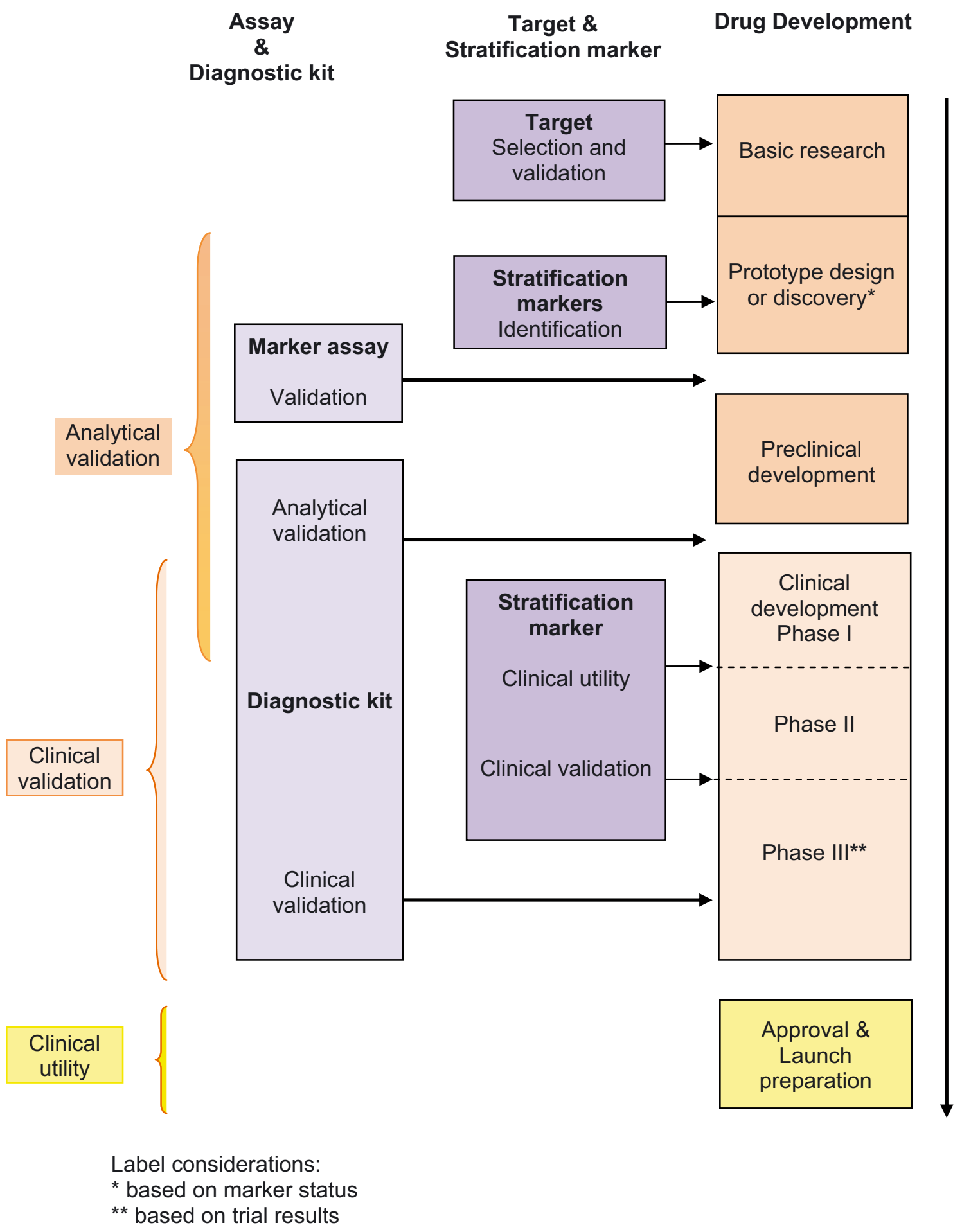

Fig. 2. The various stages in the development of a drug combined with that of a biomarker (adapted from ${ }^{[7]}$ ). 
Whether the development of a test is industrial or not and according to the status of intellectual property, several measures are recommended thereafter:

- promote good practices for the analytical development;

- promulgate recommendations for validation/qualification of the technique used to measure the biomarker;

- obtain the scientific input of regulatory agencies (EMEA, Afssaps) on the pertinence and scientific relevance of the upstream biomarker development of the couple "test/drug";

- promote the access to and the use of biological samples from clinical, academical and/or industrial studies;

- and encourage a coupling with the clinical data.

Therefore, a legal framework is required. The roundtable discussion $\mathrm{N}^{\circ} 7$ of the XXIV Giens meeting focused on this point.

It is also necessary to promote studies by academics for proof of concept, possibly in coordination with industry, in order to validate new biomarkers applicable to drugs already on the market (office of National Research Agency, "Programme Hospitalier de Recherche Clinique", Institut National du Cancer, INSERM, and the Directorate of Hospitalisation and Organisation of Healthcare).

Methodological bottlenecks will have to be removed concerning the assessment of biomarkers for target populations of restricted size and for post marketing studies. ${ }^{[18]}$

Development efforts of theranostics might enhance its value especially in case of improved efficacy or better tolerance in a target population (e.g., through the Improvement of Medical Services Rendered).

Finally, it should involve patients implicated in the search of biomarkers, particularly for the evaluation of niche indications (such as for orphan drugs).

The task force makes recommendations to allow synchronization being easier at all stages of the development of the couple "drug-diagnostic test", and to allow their disposal simultaneously.

\subsection{Recommendation for analytical validation of the test and metrological aspects}

We will neither revisit the good practice of analytical development, nor re-examine the implementation of recommendations for validating and qualifying the technique of measure of a biomarker as mentioned above. Of note, in the future, diagnostic tests will rely increasingly on innovative technologies that are evolving continuously, whether based on genetics (SNP's), transcriptomics (multiplex RT PCR), proteomics (Elisa), or imaging.

We recommend a pre-analytical validation and standardization of the biological resource and to follow either the European best practice guidelines or the current recommendations for diagnostic tests according to their type be it genetics, imaging, pharmacokinetics or pathology.

It is necessary to define validation criteria for the innovative tests.

In case a diagnostic test has an identified "ownership" without any kit available on the market, it is recommended that the technical properties of the test be published, according to the EC marking instructions.

\subsection{Authorization process for the couple drug+test}

The group recommends that a new regulatory process is put into action for the couple "drug+test" with a coupled marketing authorization and homologation (EC marking), and to initiate a synchronized procedure for market entry in order to provide a joint disposal of both the drug and its companion test. All actors should be accountable for this objective. On applying for marketing approval for the drug, 3 possible scenarii were identified for the diagnostic test: either the commercial test is already on the market (EC marking), or the test owned by an industrial is not yet on the market, or it is an in-house test and not subject to EC marking.

The recommendations for the couple "drug - diagnostic test" are as follows:

- the marketing authorization form of the drug should mention the characteristics of the test, its validity and the scientific proof of its relevance;

- should the test be marketed for the targeted indication, it should be EC marked and it should it be clearly defined in the Summary of Product Characteristics (SPC) and should be mentioned in the clinical dossier prepared for the process of approval;

- provision of a service for a test designed in industry but not available on the market might occur. It corresponds to the implementation of a test not available on the market but which should nevertheless be consistent with the CE Marking Directive, or be treated as a medical biological activity.

\subsection{Recommendations for a joint process of refunding}

The process to place a biological (or pathological) procedure on the reimbursement list is a long and complex one (figure 3 ).

The application process for a new procedure that has to be evaluated by the French National Authority for Health (HAS) is long and can last up to a year - 6 months plus an additional period of 6 months or more when complementary information is needed. 


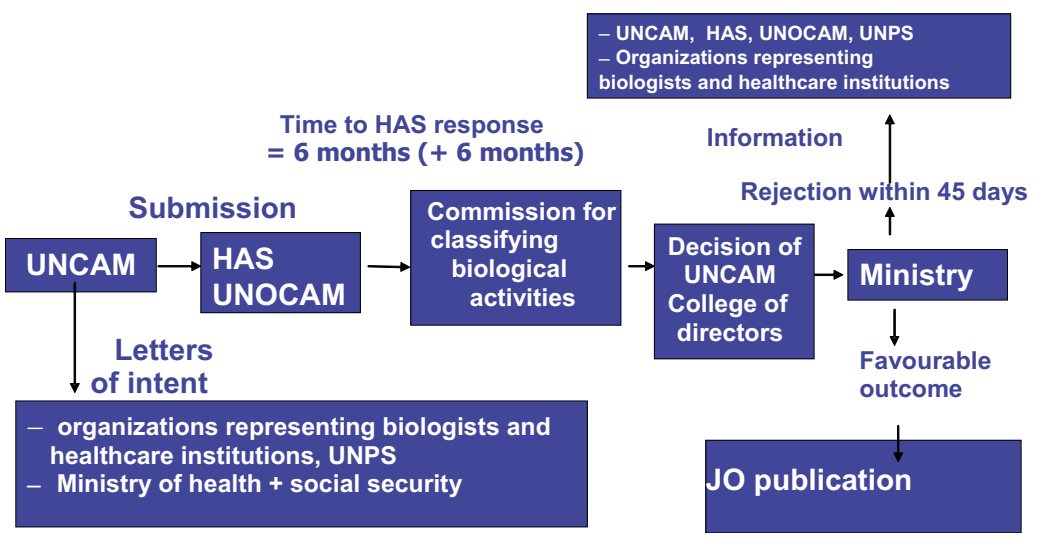

Fig. 3. Cycle to obtain inscription of a biological act on the list of refundable acts and services.

UNPS: Union Nationale des Professionnels de Santé (National Union of Health Professionals); HAS: Haute Autorité de Santé (French National Authority of Health); UNCAM: Union Nationale des Caisses d'Assurance Maladie (National Union of Health Insurance Funds); UNOCAM: Union Nationale des Organismes Complémentaires d'Assurance Maladie (National Union of Supplementary Health Insurance Insitutions); JO: Journal Officiel (Official Journal).

Once at the HAS, the evaluation of the rendered medical services of the drug is submitted to the Transparency Commission on the one hand, and on the other hand the evaluation of the expected medical services of the test is submitted to the Evaluation Commission for Professional Activities. We recommend that the application is submitted simultaneously to both Commissions in order to shorten the assessment period.

It is necessary that the application process for reimbursement should be simplified to allow similar times for assessment to ensure a simultaneous release on the market, and product availability on a large front.

We are in favour of an amendment of the wording in the social security context to assure a simplification of the registration process for reimbursement of tests.

At the same time we propose that a specific budget for innovative tests is taken into consideration in anticipation of the inscription in the nomenclature of the test. Article 51 of 2009 addressing the law on social security funding introduces a possibility of financial support for innovative actions through earmark funding ("Missions d'Intérêt Général et d'Aide à la Contractualisation", MIGAC).

\subsection{Recommendations on reporting on the couple "drug and test " and on quality assurance}

Spreading of information on the product (drug-test) and training consultants and medical technologists would be an important aspect to achieve. It could be done through a national programme based on quality assurance together with the centres of reference associated with the definition of accreditation criteria for the reference platforms and the laboratories that will actually perform the tests. This will necessitate a process of assembling the responsible people from the pharmaceutical industry with the academicals

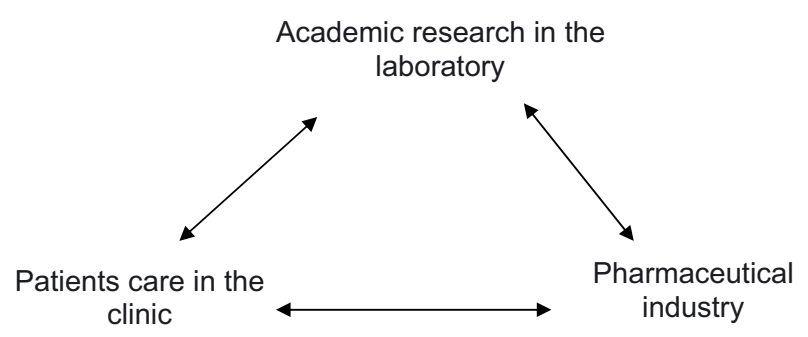

Fig. 4. Interaction between academical research, pharmaceutical industry and clinical practice.

and institutional worlds. The respective roles remain to be defined within the translational research that links basic research to clinical research (figure 4).

It is important to promote the supply of information as well as the training of consultants so that professionals be appropriately informed and respectful of the indications of prescribing the couple test/drug.

When a new combination of test/drug reaches the market, an organization capable of performing the tests respecting conditions of high quality for all involved patients, should be in place beforehand. Depending on the number of tests per year and depending on the technical and specialized skills required to perform them, reference platforms might be implicated, the accreditation criteria of which will require to be defined.

It is further necessary that the use of the tests and preparation of the samples should be harmonized. This can be achieved through a national programme of quality assurance in cooperation with reference centres or the laboratories responsible for performing the actual tests. On this level it will be necessary to train medico-technical personnel. 
This all requires a coordinated effort from the pharmaceutical industry and the academicals and institutional worlds. Each one's role is still to be defined.

Finally, it is necessary to ensure an effective transfer of technology between the laboratory using a given test and its extension to a widespread audience.

\section{Conclusion}

Theranostics promise a significant step forward for the use of more personalized medicine, better tailored for patients in terms of efficacy and tolerance. However, the development process of theranostics is complex. It is particularly important to ensure a proper phasing of all stages from development to reimbursement, through a collaborative and controlled approach.

The task force's recommendations should form the basis for reflection and action amongst the different players in the field of the development of theranostics.

As a matter of fact, all stages of the system meeting the heterogeneous and asynchronous rules should be harmonized to allow for simultaneous availability of and access to the drug and test.

Nevertheless, it is necessary to simplify the regulations to enable a better adaptation of theranostics to the speed of innovation.

Acknowledgements. Samia Rahal is acknowledged for her skilful help and Jérôme d'Enfert for his kind agreement to control the preparation process of the manuscript.

\section{Participants}

Olivier Arnaud (INSERM Tranfert), Frédéric Bibeau (CRLC Val D’Aurelle, Montpellier), Dominic Cellier (Merck Santé), Jérôme d'Enfert (Laboratoires Roche), Frédéric Eberlé (Roche Diagnostics Meylan), Agnès Goestchel (GlaxoSmithKline), JeanMarc Grognet (Ministère de l'économie, des finances et de l'emploi), Catherine Janus (Sanofi Aventis), Behrouz KassaïKoupaï (EPICIM, Lyon), Pierre Laurent-Puig (Hôpital Européen Georges Pompidou, Paris), Pascale Maisonneuve (Afssaps), Frédérique Nowak (INCa), Gilles Paintaud (CHRU de Tours), Laurence Robba (CNAMTS).

\section{References}

1. Biomarkers Definitions Working Group. Biomarkers and surrogate endpoints: preferred definitions and conceptual framework. Clin Pharmacol Ther 2001; 69(3): 89-95

2. Vogel CL, Cobleigh MA, Tripathy D, et al. Efficacy and safety of trastuzumab as a single agent in first-line treatment of HER2-overexpressing metastatic breast cancer. J Clin Oncol 2002; 20(3): 719-26
3. Slamon DJ, Leyland-Jones B, Shak S, et al. Use of chemotherapy plus a monoclonal antibody against HER2 for metastatic breast cancer that overexpresses HER2. N Engl J Med 2001; 344 (11): 783-92

4. Marty M, Cognetti F, Maraninchi D, et al. Randomized phase II trial of the efficacy and safety of trastuzumab combined with docetaxel in patients with human epidermal growth factor receptor 2-positive metastatic breast cancer administered as first-line treatment: the M77001 study group. J Clin Oncol 2005; 23 (19): 4265-74

5. Piccart-Gebhart MJ, Procter M, Leyland-Jones B, et al. Trastuzumab after adjuvant chemotherapy in HER2-positive breast cancer. N Engl J Med 2005; 353 (16): 1659-72

6. Penault-Llorca F, Balaton A, Sabourin JC, et al. Immunochemistry evaluation of HER2 status in infiltration breast cancer: technical protocol and interpretation guidelines. Ann Pathol 2002; 22 (2): 150-7

7. Phillips AK, Van Bebber S, Isssa AM. Diagnostics and biomarker development: priming the pipeline. Nature Reviews Drug Disccovery 2006; 5: 463-69

8. Lievre A, Bachet JB, Le Corre D, et al. KRAS mutation status is predictive of response to cetuximab therapy in colorectal cancer. Cancer Res 2006; 66 (8): 3992-5

9. Amado RG, Wolf M, Peeters M, et al. Wild-type KRAS is required for panitumumab efficacy in patients with metastatic colorectal cancer. J Clin Oncol 2008; 26 (10): 1626-34

10. Van Cutsem E, Lang I, D'haens G, et al. KRAS status and efficacy in the firstline treatment of patients with metastatic colorectal cancer $(\mathrm{mCRC})$ treated with FOLFIRI with or without cetuximab: the CRYSTAL experience. Journal of Clinical Oncology, 2008 ASCO Annual Meeting Proceedings (Post-Meeting Edition). Vol 26, No 15S (May 20 Supplement), 2008: 2

11. Karapetis CS, Khambata-Ford S, Jonker DJ, et al. K-ras mutations and benefit from cetuximab in advanced colorectal cancer. N Engl J Med 2008; 359 (17): 1757-6.

12. Linardou H, Dahabreh IJ, Kanaloupiti D, et al. Assessment of somatic k-RAS mutations as a mechanism associated with resistance to EGFR-targeted agents: a systematic review and meta-analysis of studies in advanced nonsmall-cell lung cancer and metastatic colorectal cancer. Lancet Oncol 2008; 9 (10): $962-72$

13. Picard S, Titier K, Etienne G, et al. Trough imatinib plasma levels are associated with both cytogenetic and molecular responses to standard-dose imatinib in chronic myeloid leukemia. Blood 2007; 109 (8): 3496-9

14. Tuma RS. Disease progression in some cancers may be due to low blood levels of targeted therapies. J Natl Cancer Inst 2008; 100 (13): 912-3

15. Maloney DG, Grillo-Lopez AJ, White CA, et al. IDEC-C2B8 (Rituximab) anti-CD20 monoclonal antibody therapy in patients with relapsed lowgrade non-Hodgkin's lymphoma. Blood 1997; 90 (6): 2188-95

16. Igarashi T, Kobayashi Y, Ogura M, et al. Factors affecting toxicity, response and progression-free survival in relapsed patients with indolent B-cell lymphoma and mantle cell lymphoma treated with rituximab: a Japanese phase II study. Ann Oncol 2002; 13 (6): 928-43

17. Chung $\mathrm{CH}$, Mirakhur B, Chan E, et al. Cetuximab-induced anaphylaxis and IgE specific for galactose-alpha-1,3-galactose. N Engl J Med 2008; 358 (11): 1109-17

18. Vray M, Girault D, Hoog-Labouret N, et al. Methodology for small clinical trials. Therapie 2004; 59 (3): 281-6

Correspondence and offprints: Paul Landais, Hôpital Necker Enfants Malades, Service de Biostatistique, 149 rue de Sèvres, 75006 Paris, France. E-mail: landais@necker.fr

Valérie Méresse, F Hoffmann-La Roche Ltd, Steinentorberg 12, 4070 Basel Suisse. E-mail: valerie.meresse@roche.com 